\title{
Quality-Preserving Image Downsizing
}

Matthew Trentacoste

\author{
Rafal Mantiuk \\ University of British Columbia
}

\author{
Wolfgang Heidrich
}

\section{Introduction}

The image quality of a digital viewfinder is considerably lower than that of a through-the-lens optical system. While the sensor may be capable of capturing 10 or 20 megapixels, the screen of the viewfinder is typically constrained to resolutions under 1 megapixel. The limited resolution makes it impossible to discern all the small details of the captured image. Small blurs and noise that are present in the full-size image can render the image unusable for certain tasks, yet these artifacts may be too small to be discernible in the downsampled version shown on the camera viewfinder.

We preserve spatial detail such as blur and noise while creating the thumbnail image for presentation in a digital viewfinder. We present an efficient method for enhancing the artifacts that may be present in both still images and live preview video streams, making them more identifiable, and thus decreasing the chance of capturing low-quality images. We analyze the image to detect artifacts present but too small to be visible on a digital viewfinder, and modify the downsampled thumbnail image such that they are large enough to be discernible, as seen in Figure 1. The result more accurately reflects the original image, allowing faster and more accurate assessments of quality in the field or when browsing thumbnails.

Our blur enhancement produces an image with out-of-focus regions further blurred like Bae and Durand [2007], but it produces a lower resolution approximation at significantly reduced computational cost. Similar to our work, Samadani et al. [2010] developed a method for amplifying specific artifacts present in full-size images to be visible in thumbnail images. However, their method cannot accurately detect motion blur or noise in textured areas.
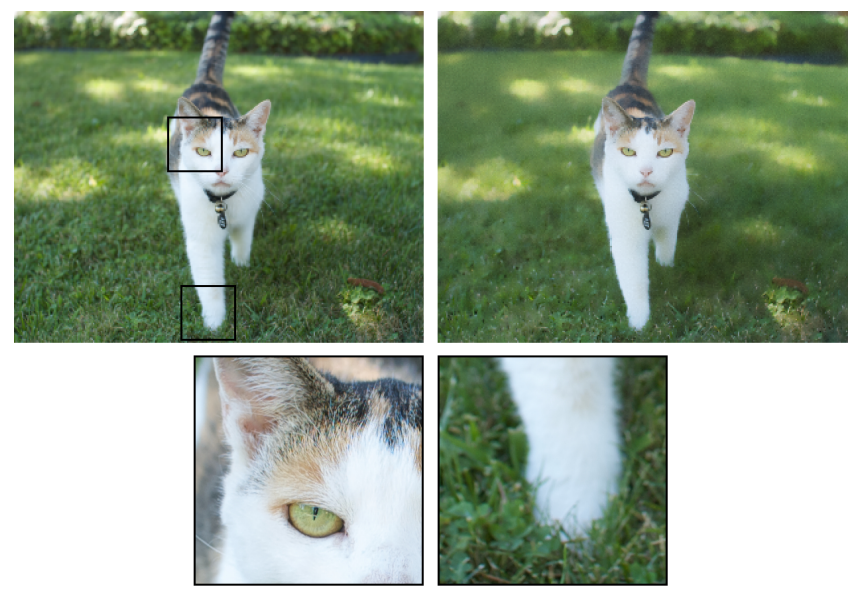

Figure 1: A comparison of our quality-preserving downsampling algorithm (right) with a regular thumbnail (left). The full resolution original image is shown in the cropped inserts below. Note that the cat's face is the only part of the image that is in focus (see detail regions below), a feature that is preserved by our algorithm.

\section{Our approach}

In order to make the blur and noise artifacts more apparent in a downsampled thumbnail image, we first must analyze the full-size image to determine how much of each attribute is present. Given an estimate of the significance of both blur and noise, we amplify these characteristics so they remain visible in the generated thumbnail. This process is particularly important when the strength of an artifact is large enough to be of visual significance, but small enough to be invisible in the thumbnail.

The outline of our algorithm is as follows: 1) We identify the strength of both blur and noise present in the full-size image. 2) Based on our blur estimate, we determine how much blur needs to be added to each region of the thumbnail. 3) We downsample the image, and add the required amount of blur. 4) For each image region, we model how much of the original noise is lost due to both the downsampling operation and the subsequent blur enhancement. 5) Finally, we re-introduce the required amount of noise for each pixel. Representations of the intermediate steps can be seen in Figure 2 .

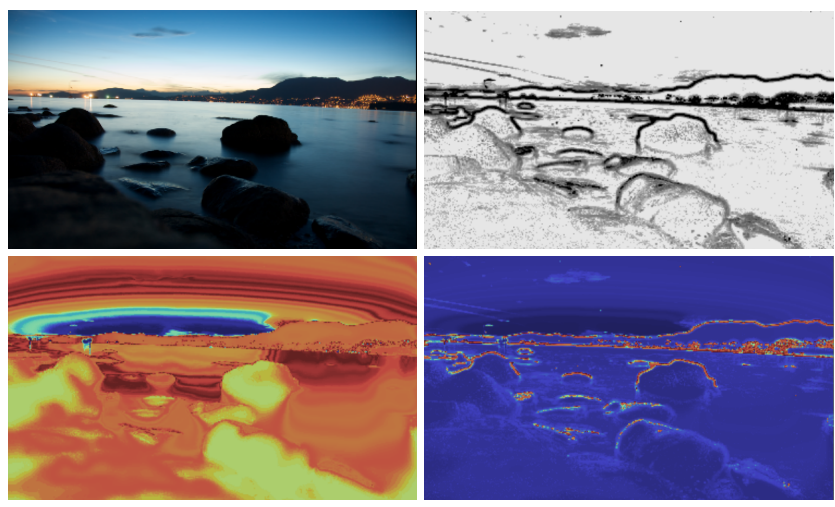

Figure 2: Upper left: original image. Upper right: estimated blur. Lower left: false color noise map. Lower right: false color bluradjusted noise map.

In cases like defocus and motion blur, the amount of blur typically varies across the image. In order to retain the detail of in-focus regions while further removing detail in out-of-focus regions, our blur estimation routine determines a spatially-variant estimate of blur across an image. Similarly, our noise estimation reliably estimates the noise present at different pixel intensities, and compensates for the differing amount of noise reduction resulting from the increased blur of the thumbnail.

The end product is a computationally effective means of preserving small-scale image artifacts in downsampled images that ensures they are visible on a digital viewfinder or when browsing image thumbnails.

\section{References}

Bae, S., And Durand, F. 2007. Defocus magnification. Computer Graphics Forum 26, 3, 571-579.

SAMAdAni, R., MaUer, T. A., Berfanger, D. M., AND CLARK, J. H. 2010. Image thumbnails that represent blur and noise. IEEE Transactions on Image Processing 19, 2, 363-373. 